\title{
Interactive comment on "The effects of decomposing invasive jellyfish on biogeochemical fluxes and microbial dynamics in an ultraoligotrophic sea" by Tamar Guy-Haim et al.
}

\section{Anonymous Referee \#1}

Received and published: 21 July 2020

The manuscript describes changes in nutrients and microbial communities in a laboratory based jellyfish decomposition experiment. The manuscript is well written, the subject area is of interest and the particularly the biodiversity aspect is novel. However, the authors need to to take more account of the incubation system used more into account for the presentation and discussion of the data. Firstly, there is an evolution of processes during decomposition resulting from colonisation of the biomass, microbial growth dynamics and the sequential nature of the decomposition of particulate organic matter. Secondly in the discussion the limitations of the incubation method which resulted in large changes in conditions and in particular oxygen concentrations needs to 
In the abstract impacts on phytoplankton are mentioned, but there is no discussion of possible links between bloom decomposition and phytoplankton community structure and production in the introduction 33-45. As well as providing a food source to scavenging fauna, the presence of jellyfish carcasses on the sediment surface also simultaneously blocks oxygen transfer to the underlying sediment and stimulate anaerobic respiration processes, resulting in sediment reduction and accumulation of toxic sulphides (See cited Chelsky et al paper). These changes in sediment conditions result in migration or mortality of infauna, which are in turn a major influence on nutrient cycling (See for example Welsh 2000 Chemistry \& Ecology 19, 321-342; Stief 2013 Biogeosciences 10, 2829-46 for reviews). These potential negative effects on benthic fauna and the indirect effect this has on nutrient cycling deserve a mention here, especially since they are again mentioned in the abstract.

65. This biomass addition is equivalent to approx. $3.5 \mathrm{~kg}$ per square metre. How realistic is this for a natural bloom collapse in the study area.

70-80. Were the cores incubated under light or dark conditions i.e. are there any effects of photoautotrophic activity on oxygen and nutrient concentrations

90-95. There are several issues with using this equation to calculate average fluxes over the entire incubation period as done in the results. Firstly, the equation assumes that the change in concentration is linear (consistent flux rate), but as the figure shows this is not true and fluxes rates evolve over time, as would be expected during decomposition (see cited decomposition studies), and in some cases reverse direction. At least in some cases, this impact could be minimised by calculating between time points, when conc changes would be closer to linear and changes between periods would show the evolution of flux rates over time. Secondly, fluxes are largely due to diffusion and diffusion rates depend on the concentration gradient between the sediment porewater and the overlying water. Therefore in a closed system like the one

Printer-friendly version

Discussion paper 
used here, the changes in water column solute concentrations caused by the fluxes inhibit the rate of the flux that creates them by decreasing the concentration gradient between the sediment and water. This is especially true for oxygen where the water column conc falls to zero i.e. there is no oxygen consumption at the end of the experiment because there is no oxygen demand, but because there is no oxygen to supply the oxygen demand. Thirdly, as the extremely large change in water column oxygen concentration and therefore fluxes, aerobic processes become increasingly inhibited over time causing a shift to anaerobic processes, which would impact both nutrient dynamics and microbial community composition.

121-127. Presumably the $1.7 \mathrm{~mL}$ incubated refers to the seawater in the cores. However, it would be expected that the bulk of bacterial production would occur associated with the jellyfish tissues and the sediment in contact with these.

129-144. As above, this is not measuring the overall changes in populations, just those in the water column.

145-160. What statistical analyses were performed on the oxygen and nutrient data.

160-190. As above the effects of decomposition processes evolve over time due to colonisation processes, the sequential nature of decomposition e.g. PON decomposed to DON and DON to ammonium, shifting conditions and ultimately depletion of the biomass. This is shown by the non-linearity of the concentration changes that show that the production/consumption processes causing the fluxes are changing with in some cases the flux changing direction. Therefore, data need to be analysed in a manner that shows these shifting rates and the changing nutrient ratios they produce. It would also be useful to indicate what fraction of the $C, N \& P$ in the added biomass were actually mineralised over the course of the experiment. Especially as the data in the figure indicate that the decomposition rate had not even peaked by the end of the experiment, as ammonium production rates were still increasing at the end of the experiment. Indeed the highest rate of oxygen demand was at the end of the

Printer-friendly version

Discussion paper 
experiment, despite low water column concentration present at this time.

204-208. There is no description of the sediment analyses in the methods section.

4.1. This section would be much improved by reanalysing the oxygen and nutrient flux with time. This would show how these evolved over time and how the composition of the TDN and TDP fluxes shifted over time. This would allow discussion of the decomposition process e.g. leaching versus decomposition, sequential mineralisation etc. Also some data on the proportion of particularly the $\mathrm{N}$ and $\mathrm{P}$ present in the biomass that was actually mineralised during the experiment would be useful, as it appears the decomposition process was only partially completed, so overall effects would be greater over longer time periods. Finally, some context needs to be given when making comparisons to the natural system e.g. how does the biomass density compare? How does a closed system with a $40 \mathrm{~cm}$ water column compare to in situ conditions with a large water column (greater oxygen available), which can be resupplied by water movements such as currents and exchange with the atmosphere i.e. potential in situ effects would be very, very much lower than those measured.

275-278. This $\mathrm{N}: \mathrm{P}$ ratio is incorrect. It is not a \%:\% (weight:weight) ratio, it is an atom:atom (Mol:Mol) ratio. Therefore, the weights of $\mathrm{N}$ and $\mathrm{P}$ need to be divided by the atomic masses of $\mathrm{N} \& \mathrm{P}$ and the ratio of these compared.

317-324. Growth efficiency also depends on the type of respiration and decreases in the order of aerobic Approx. 0.5) > nitrate reduction > metal reductions > sulfate reduction (.0.2). Therefore, fixed production does not equal fixed rate of respiration as the type of respiration which is taking place shifts with oxygen conditions. Such changes would be even greater in jellyfish associated biofilms and in the surface sediments (See cited paper by Chelsky et al. 2016, which shows a shift to iron and sulfate reduction in the sediment in situ). The shift in your nitrate data from production (net nitrification) to consumption (net nitrate reduction), demonstrate this shift in dominance from aerobic to anaerobic processes in the benthos. Whereas, the water column effect in situ is

Printer-friendly version

Discussion paper 
likely very, very different from thr changes that occurred in your cores.

317-361. Again the shifting conditions within the cores need to be discussed. As above there would be a shift from aerobic to anaerobic groups over time as conditions shifted. However, this would impact taxonomic diversity to a greater degree than functional diversity e.g. increased availability of protein would select for proteolytic bacteria, but oxygen availability would impact whether these were aerobic or anaerobic taxa of proteolytic bacteria.

Interactive comment on Biogeosciences Discuss., https://doi.org/10.5194/bg-2020-226, 2020. 